\title{
Rare sequelae of ectopic gestation: A case report of lithopaedion
}

\author{
${ }^{1}$ I A Ekanem, ${ }^{2}$ E I Ekanem, ${ }^{1} \mathrm{C}$ C Chukwuegbo, ${ }^{1} \mathrm{~T}$ I Ugbem, ${ }^{2}$ A D Ekanem \\ ${ }^{1}$ Department of Pathology, University of Calabar Teaching Hospital, Calabar, Nigeria \\ ${ }^{2}$ Department of Obstetrics \& Gynaecology, University of Calabar Teaching Hospital, Calabar, Nigeria
}

\begin{abstract}
Lithopaedion is a rare clinical condition resulting from dystrophic calcification of extra-uterine foetus after demise. It often involves singleton foetuses but twin lithopaedions have also been reported.

This case report is on a 30-year old Para ${ }^{1+4}$ woman who presented with a 5-year history of abdominal discomfort and swelling. She became pregnant about 6-years ago and attended clinics only thrice. However at the $9^{\text {th }}$ month, she developed a fever and thereafter no longer felt foetal movements for which she took herbal concoctions. Pregnancy symptoms subsequently ceased and menstruation resumed 6 months later. She had laboratory and radiologic investigations and then laparotomy with extraction of a stone baby. She made good post-operative recovery.
\end{abstract}

Keywords: Lithopaedion, ectopic gestation, dystrophic calcification, mock labour, laparotomy

\section{Introduction}

Lithopaedion is defined as calcified product of ectopic gestation. It is due to dystrophic calcification after foetal death. It is a rare occurrence seen in only about $1.5 \%-1.8 \%$ of abdominal pregnancies. Put in perspective, abdominal pregnancy incidence is about one in 11,000 pregnancies. Most cases involve singleton foetuses but twin lithopaedions have also been reported[1], [2].

\section{Case Report}

The patient is a 30-year old Para ${ }^{1+4}$ woman who presented with a 5-year history of abdominal discomfort and swelling. Prior to this problem, she became pregnant about 6 -years ago and commenced antenatal visits at 3 months gestation. Altogether, she had 3 visits with the last at the $5^{\text {th }}$ month. However, at the $9^{\text {th }}$ month she developed a fever; thereafter no longer felt foetal movements for which she took herbal concoctions. She then noted a regression of symptoms of pregnancy and 6 months later she started menstruating.

Abdomino-pelvic ultrasonographic scan showed a peritoneal cavity virtually obscured by dense acoustic shadows with calcified vertebral column and half-moon shaped skull. Abdominal X-ray (see Fig. 1) showed a lower abdominal mass lying transversely and having a calcified vertebral column and shell. She had laparotomy(see Fig. 2) with extraction of a stone baby and made good post-operative recovery.

\section{Post Mortem Findings}

Post mortem examination shows a large calcified baby with the skull and right upper and lower limbs buried into the torso (see Fig. 3). The left upper and lower limbs are flexed and partly fused with the trunk. The mass weighs $3.5 \mathrm{~kg}$ and is covered by brownish calcific shell. The outline of digits on the right reveals normal number of digits. Cut section into the mass (see Fig. 4) shows the vertebral column, right humerus, autolysed muscles and internal organs as well as inspissated greenish material (meconium) inside autolysed gut.

Microscopic examination of the tissues shows skin, hair follicles and subcutaneous adipose tissues as well as acellular outline of skeletal muscle bundle surrounded by fibrocollagenous material.

\section{Discussion}

This index case presented because of vague discomfort but patient was more concerned about the abdominal swelling. Her history is typical with mock labour and later cessation of pregnancy signs. The occurrence of fever about the time of foetal demise could be due to coincidental infection or malaria. The fever might not have been the direct cause of the foetal death as this was almost inevitable considering that it was an abdominal pregnancy and the patient did not present in a health institution for care at term. The possible toxic effects of unknown herbal preparation could have hastened foetal demise.

As a rule, the formation of lithopaedion takes place only if the foetus remained sterile. In addition, the foetus must be extra-uterine, survive for longer than three months and the necessary conditions for deposition of calcium in the tissues must be present especially sluggish local circulation[3]. The discovery after five years is consistent with previous reports. Previous reports revealed foetal retention of more than 55 years [4].

On the basis of site of calcium deposition, lithopaedion can be subclassified into three viz (i)Lithokelyphos where only the foetal membranes are calcified and not adherent to the foetus. The foetus may be unchanged or 
mummified. This type is thought to be due to the membranes remaining intact after foetal death, (ii) Lithokelyphopaedion where both the membranes and foetus are calcified and are adherent to one another. It is believed to be the result of absorption or escape of amniotic fluid after foetal demise. This is probably the most common type. (iii) Lithotecnon (true lithopaedion) where only the foetus is calcified. This type is probably due to escape of the foetus into the abdominal cavity, leaving its membranes behind[3]. The present case is a true lithopaedion.

\section{Figures}
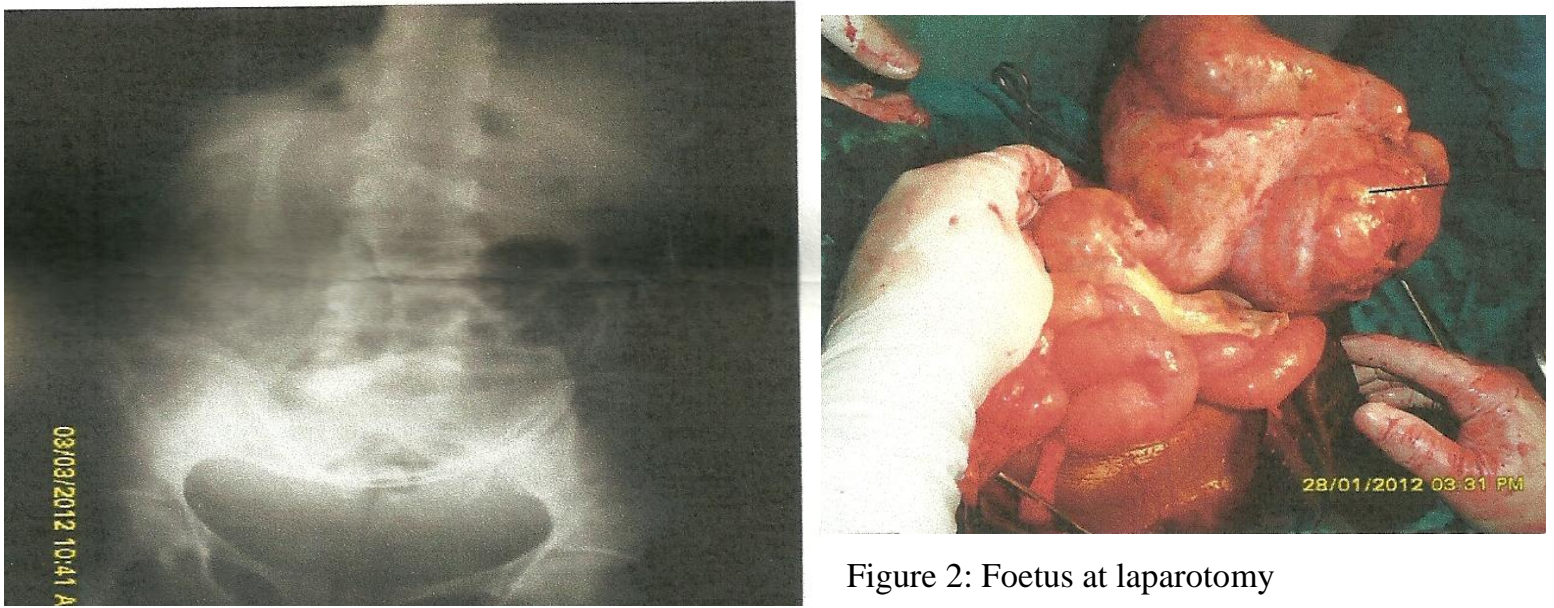

Figure 2: Foetus at laparotomy

Figure 1: Plain Chest X-ray shows the foetus in longitudinal lie with calcific outline
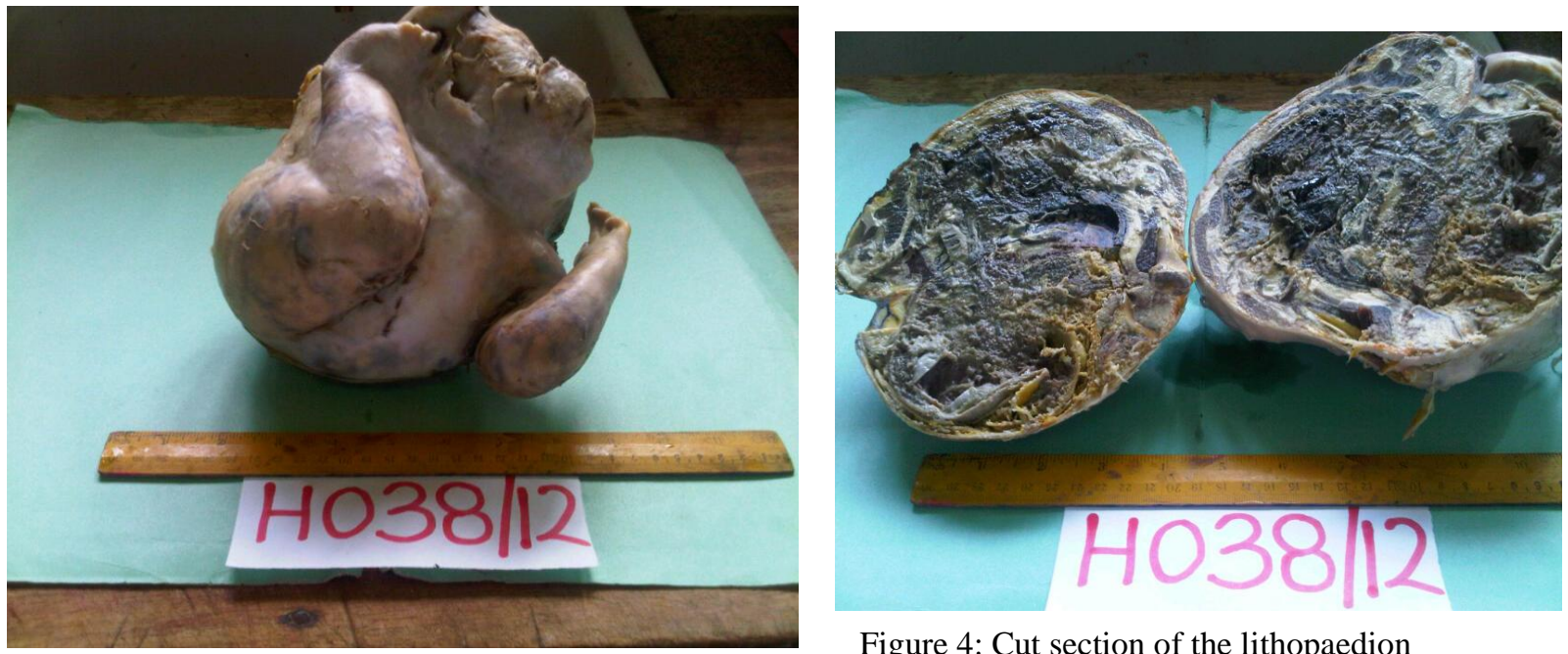

Figure 4: Cut section of the lithopaedion

Figure 3: Gross appearance of the lithopaedion

\section{CONCLUSION}

Lithopaedion usually presents as a "mock labour" followed by regression of pregnancy signs and symptoms with eventual return of menstruation and even subsequent uneventful pregnancies[5]. This was the case with the present case. Lithopaedion may not come to clinical notice. When symptomatic, lithopaedion is known to cause compression and rupture of adjacent viscera, intestinal obstruction as well as obstructed labour[6],[7]. It is often discovered accidentally during clinical examination, radiologic investigation, or at autopsy[1], [8]. Some cases have been discovered nearly sixty years after foetal demise[4], [5]. 


\section{REFERENCES}

[1] J. M. Mishra, T. K. Behera, B. K. Panda, and K. Sarangi, “Twin lithopaedions: a rare entity.," Singapore medical journal, 48( 9), 2007, 866-8

[2] J. K. Roberts, “A lithopaedion in twins.," BMJ, 1952, 130

[3] P. Cave, "Note on Lithopaedion with report of a case.," BMJ, 1, 1937, 383-85

[4] "Lithopedion - Wikipedia, the free encyclopedia." [Online]. Available: http://en.wikipedia.org/wiki/Lithopedion. [Accessed: 07-Mar2013].

[5] J. B. Fraser, "Lithopaedion," BMJ, 1913, 1624-25

[6] C. C. Ekwempu, "Obstructed labour due to tubal lithopaedion: a case report," East Afr Med J, 54( 6), 1977, 353-5

[7] I. S. A. Zaheer, “Acute intestinal obstruction caused by lithopaedion,” British Journal of Surgery, 58( 5), 1971, 401-402

[8] P. Noble, "Abdominal pregnancy and lithopaedion,” Journal of the Royal Society of Medicine, 96, 2003, 423 